\title{
Encyclopedia
}

\section{Role of Impedance Based Cellular Assays in the Hepatology Research}

Created by: Katie Morgan

Revised by: Nicole Yin

Version received: 15 January 2020

The development of organ on a chip technology utilising human cells, has the advantages of replacing animal experimentation and providing data more applicable to study of human cell biochemistry and disease. However, measurement of metabolites often requires cell lysis permitting only a single end-point measurement. Development of non-destructive methods for monitoring cell cultures in real-time is desirable in helping to inform pathways of disease and define targets for pharmacological intervention. Impedance-based cellular assays, representing one such technique, are designed to monitor cellular impedance, a property of the insulating nature of cell membranes, resulting from changes in cell shape caused by signalling events inside and between cells. This relies on culturing primary cells or cell lines in vitro directly on microelectrodes or using electrodes in culture media and then monitoring changes in impedance over time in response to external treatments compared to untreated controls. Impedance measurements are recorded in real-time at one or several frequencies and can be sampled several times a second for as long as desired. This is beneficial in pinpointing changes with time in culture and can be useful in determining the starting point of a toxic effect or a therapeutic window. A further advantage of impedance-based cellular assays lie in their ability to monitor the cell culture without using specific cell or protein labels, which might otherwise influence the target being studied. Depending on the system used, changes in impedance can be correlated with alterations in basolateral adhesion, membrane integrity, tight junctions and barrier function.

\section{Impedance based cellular assays in hepatology}

The development of organ on a chip technology utilising human cells, has the advantages of replacing animal experimentation and providing data more applicable to study of human cell biochemistry and disease. However, measurement of metabolites often requires cell lysis permitting only a single end-point measurement. Development of non-destructive methods for monitoring cell cultures in real-time is desirable in helping to inform pathways of disease and define targets for pharmacological intervention. Impedance-based cellular assays, representing one such technique, are designed to monitor cellular impedance, a property of the insulating nature of cell membranes, resulting from changes in cell shape caused by signalling events inside and between cells. This relies on culturing primary cells or cell lines in vitro directly on microelectrodes or using electrodes in culture media and then monitoring changes in impedance over time in response to external treatments compared to untreated controls. Impedance measurements are recorded in real-time at one or several frequencies and can be sampled several times a second for as long as desired. This is beneficial in pinpointing changes with time in culture and can be useful in determining the starting point of a toxic effect or a therapeutic window. A further advantage of impedance-based cellular assays lie in their ability to monitor the cell culture without using specific cell or protein labels, which might otherwise influence the target being studied. Depending on the system used, changes in impedance can be correlated with alterations in basolateral adhesion, membrane integrity, tight junctions and barrier function.

Use of impedance-based cellular assays have been reported for a variety of tissues, including cardiomyocytes, neuronal

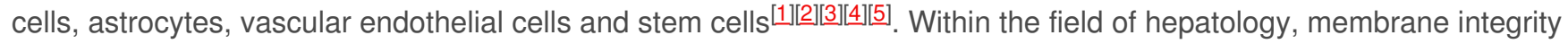
and development of cell polarity and tight junctions are all important to the function of the liver and disruption of these structures can result in hepatic pathologies. Current methods of investigating polarity and tight junction formation, such as immunohistochemistry, protein quantification or gene expression, usually require destruction of cell cultures or require replicate cultures for sampling at different time points. Methods to monitor membrane integrity and tight junctions over 
time would give more complete information on the initiation and progression of disease processes and drug toxicity mechanisms.

\section{Viral Hepatitis}

Viruses such as hepatitis B and C utilise proteins of the tight junction complex as co-receptors for entry into cells[6]. Therefore, compromised membrane integrity is of specific interest and should be monitored in pathologies such as hepatitis $\mathrm{C}$, a leading cause of hepatocellular carcinoma ${ }^{[7]}$.

One challenge to studying hepatitis $B$ and $C$ infection and antiviral therapeutics in vitro is the inability to transfect most cell lines with viruses. Two commonly used hepatic cell lines, Huh-7 and HepaRGs, have both been used extensively in the study of hepatitis B and C[8][9]10][11][12][13][14]. Huh-7 cells, established in 1982 from a human hepatocellular carcinoma[15], are known to produce the human plasma proteins albumin, transferrin, alpha fetoprotein and fibronecti146]. Their ease of transfectability and their high susceptibility to hepatitis $C$ as they promote the replication of the virus have made the development of drugs possible ${ }^{[10][15][17] . ~}$

To the best of our knowledge, no literature has been produced to date showing the use of IBCA in the study of hepatitis infection. However, Pennington and Van de Walle used the ECIS Z theta to show proof of the concept that viral infection can be studied using IBCAs. Their study centred around the feline herpes virus type 1 (FHV-1) in kidney cells and detected dose-dependent changes due to virus-induced cell death[18].

Further work with Huh-7 or HepaRG cells using IBCA may aid future in vitro studies of viral pathogens and possible therapeutic interventions within hepatology.

\section{Drug Induced Liver Injury}

There is a need for non-invasive label-free real-time read outs in drug development, as labels often mask or interfere with the molecule of interest. There are some techniques for monitoring cultures label-free, but combining this with a real-time platform can also identify the time kinetics of the toxic effect and could aid in defining dosing regimens.

Here we will discuss the advantages of IBCA alongside existing assays in a variety of cell lines.

Failure to detect early hepatotoxicity is a major cause of drug attrition. A study by Gerets et al., ${ }^{[19]}$ used the IBCA $x C E L L i g e n c e^{T M}$ system together with other techniques to better characterise current hepatic in vitro toxicity models using HepG2, HepaRG and PHH. The xCELLigence ${ }^{\text {TM }}$ Real-Time Cell Analysis (RTCA) system has been applied in studies of cell adhesion, spreading and invasion[20] barrier function[21] , cytotoxicity [22], cell signalling and co-culture studies [23][24] and more.

Gerets et al., ${ }^{[19]}$ investigated the expression levels of cytochrome P450 (CYP) activity indicative of toxicity using qRTPCR and data generated by the $x C E L L i g e n c e^{T M}$ system for any predictive correlation with hepatotoxicity. Using this system, they reported that HepG2 cells responded poorly to CYP450 inducers, which correlated with gene expression and CYP enzyme activity ${ }^{19}$. CYP was inducible under all circumstances in HepaRG and primary human hepatocytes (PHH); again, gene expression and CYP activity correlated ${ }^{[19]}$. Using standard assays and comparing with LC50 cytotoxicity curves generated from $x$ CELLigence ${ }^{T M}$ data, they showed that all cell lines were sensitive to hepatotoxins, though none at a high enough level to use in predictive experiments ${ }^{[19]}$. It is important to note here that a loss of impedance does not necessarily mean a loss of cell viability [25][26][27], but detects a change in the membrane.

In a further study from this group 28 , the effect of 5-day exposure to 50 compounds on the viability of HepG2, HepaRG, $\mathrm{PHH}$ and primary rat hepatocytes was investigated by comparing the cell index $(\mathrm{CI})$ generated from $\times C E L L i g e n c e$ 
monitoring with viability measurements based on proteases present in culture using the Promega CellTiter-Fluor assay. The viability data from the two platforms showed a correlation for HepG2 and HepaRGs, but not for PHH or primary rat hepatocytes. Other studies highlight the limitations of poor correlation between in vitro rat and human hepatocyte models $[29]$.

There is currently a lack of comparison of impedance vs. classical toxicity assays.

Atienzar et al., [28] also investigated the application of XCELLigence ${ }^{T M}$ specific real-time cell analysis to monitor the effect of calcium modulators, anti-mitotics and DNA damaging agents. They determined that it may be possible to identify specific impedance-based signatures dependent on the mechanism of action, although further work needs to be done. These studies showed an important correlation that directly relates impedance measurements to biological viability and activity.

Impedance-based assays combined with MTT, total ATP or other classical viability and metabolic assays may be important for elucidating the mechanism of drug action at a cellular level. These studies highlight the benefits of using a sensitive real-time model for drug induced liver injury and the generation of predictive values, such as LC50, alongside traditional viability assays, predictability studies and gene expression data. It also highlights the benefit of seeing in real time when damage or change occurs in the cell. This information is useful in identifying the time and mechanism of the toxic effect, planning dosing regimens, and also in planning future animal experimentation. Ultimately, knowing how and when to expect an effect could reduce the number of animals needed in drug toxicity testing.

\section{Toxicity Assays Using HepaRG Cells}

The HepaRG ${ }^{\mathrm{TM}}$ cell line is a human line that has emerged in recent years as a suitable alternative to other immortalized cell lines for toxicology studies, with function comparable to $\mathrm{PHH}$. [30] The HepaRGTM cell line was isolated from a female around age 30 with grade I differentiated hepatocellular carcinoma concurrent to hepatitis $\mathrm{C}[31$. These cells are unique in that they are a bipotent line, which can be differentiated to generate an intrinsic co-culture of hepatocytes and cholangiocyte-like cells, giving a better representation of the liver parenchyma.

Spectral karyotyping of HepaRGs ${ }^{\mathrm{TM}}$ revealed a stable and unique genotype which is consistently maintained through several passages. The cultures remained stable for 4 weeks and beyond, and retained characteristics of differentiation, making HepaRG ${ }^{\text {TM }}$ cells a viable model for drug toxicity testing. In contrast to other hepatic cell lines, fully differentiated HepaRGs ${ }^{\text {TM }}$ provide a unique co-culture of cholagiocyte-like and hepatocyte cells, making them a useful model for drug transporter studies and for use in cholestatic models [26][29][32][33].

Published work from our laboratory, measuring total impedance using ECIS technology, has shown a dose-dependent response in fully differentiated HepaRG ${ }^{T M}$ cells following exposure to the model hepatotoxin paracetamol $\left(\right.$ APAP) ${ }^{[30]}$ (Figure 1). The deconvolution of data revealed a mechanism of dose dependant APAP toxicity not previously described ${ }^{[30]}$. This is relevant to the clinical presentation of paracetamol toxicity and may inform future investigation into specific molecular targets in the development of therapeutics. 

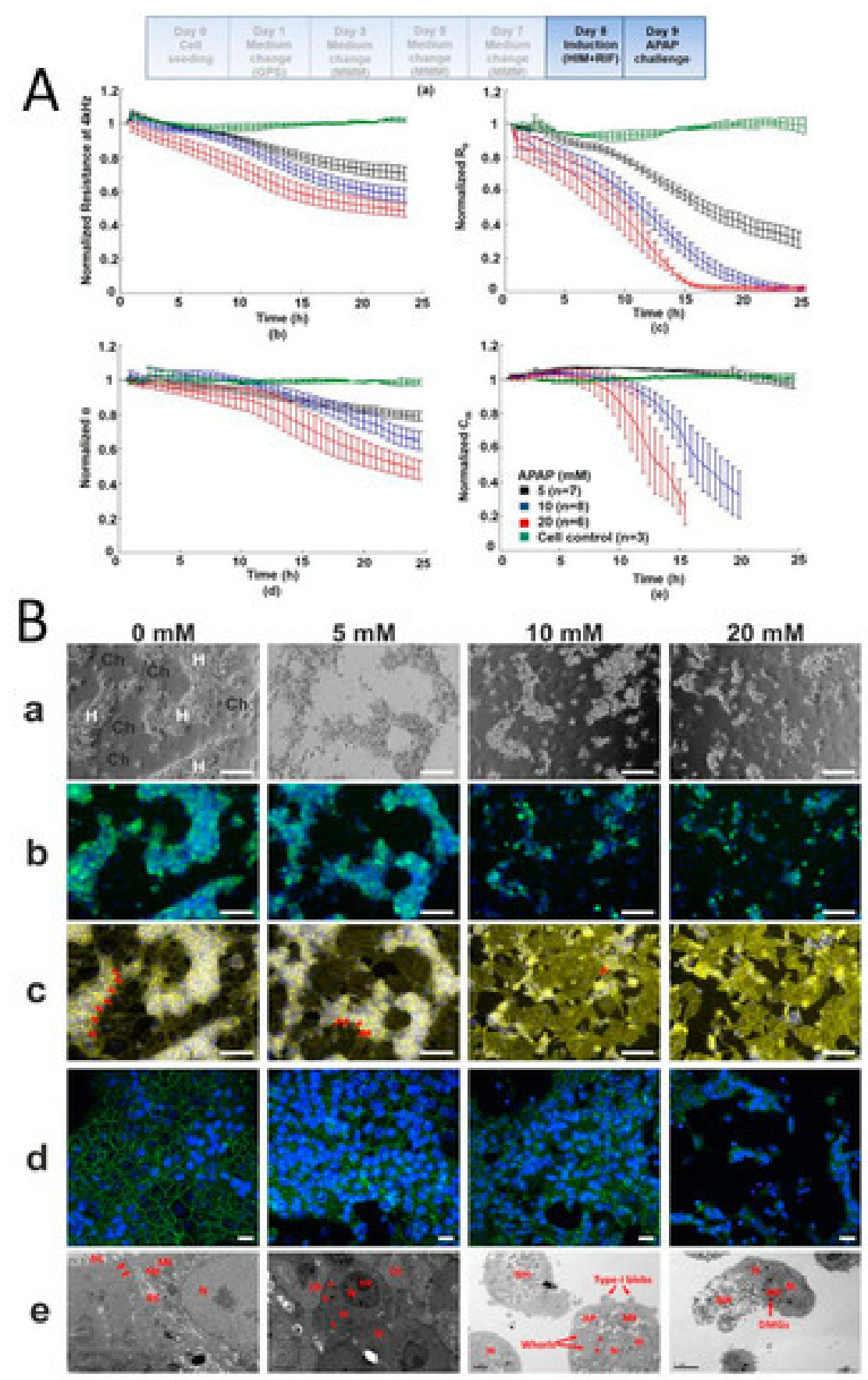

Figure 1. Direct hepatotoxicty signature of HepaRG cells treated with paracetamol; correlation between cellular impedance and immunocytostaining (A) HepaRG cells treated for $24 \mathrm{~h}$ with paracetamol: untreated cell control; $5 \mathrm{mM} ; 10$ $\mathrm{mM}$; and 20 mM. Paracetamol caused a dose-dependent decline in normalized resistance-a global indicator of cellular status. Graphs show (top left) deconvolution of total impedance, (top right) tight junctions, (bottom left) basolateral adhesion and (bottom right) membrane capacitance (B) Correlation to IBCA of confocal microscopy and TEM showing HepaRG cells' progressive loss of tight junctions with increasing paracetamol concentration. (a) phase contrast microscopy where $\mathrm{H}$ refer to hepatocytes and $\mathrm{Ch}$ to cholangiocytes (b) transferrin and Hoescht staining showing loss of function due to destruction of tight junctions (c) F-actin staining (yellow) shows breakdown of cytoskeleton with increasing paracetamol concentration. Red arrows show tight junction associated F-actin bands (d) confocal microscopy showing loss of tight junction protein ZO-1 (green) (blue nuclear staining with Hoescht) (e) representative TEM confirming loss of tight junctions and cells showing a necrotic/apoptotic appearance. Red arrows represent mitochondria and tight junctions at $0 \mathrm{mM}$, an electron dense perimeter around hepatocytes at $5 \mathrm{mM}$, type I blebbing at $10 \mathrm{mM}$ and dense mitochondrial granules at $20 \mathrm{mM}$ (Reprinted from Gamal et al., 2017) ${ }^{[30]}$.

\section{Toxicity Assays Using Pluripotent Stem Cells}


The use of human embryonic stems cells (hESC) and human induced hepatocyte-like cells derived from pluripotent stem cells (hIPS) differentiation to hepatocyte-like cells is very attractive in hepatology research. ECIS offers the advantages of providing hepatocytes from non-diseased tissue, reproducibility between experiments and IPS cells offer the opportunity to study specific disease states and genotypes known to be important in some adverse responses to drugs. Although they have already proven useful in a number of studies, further work is needed to optimise differentiation protocols to achieve mature hepatocyte phenotype and function. hESCs and hIPS could potentially generate tailored models of human liver disease and population-relevant models for drug toxicity testing [34][35][36]. They may also be of particular benefit in the assessment of drugs that cause idiosyncratic toxicity due to genetic polymorphisms.

Zhou et al., [37] showed that impedance data acquired using ECIS to monitor real-time growth kinetics as well as the differentiation of pluripotent stem cells to hepatic progenitors and hepatocyte-like cells followed established signatures of cell differentiation. These were assessed by microscopy and were measurable in real time by ECIS. The readings from the independent wells were uniform and remained in close proximity throughout the differentiation process 3 [37]. This result is important, as it demonstrates the reproducibility of differentiation between replicates.

They also used ECIS and Promega CellTiterGlo total ATP assay in parallel to assess the toxicity of compounds BMS4 and BMS5 on hESCs ${ }^{[37]}$. Using the ECIS Z theta system at a single frequency with one electrode per well showed that there was good correlation of results from the ECIS and CellTiterGlo assays. This demonstrates that through using standard methods, e.g., microscopy, gene expression, functional assays, to support impedance data, it is possible to establish an impedance signal for a particular cell culture under a particular set of conditions.

This study highlights two very important aspects of IBCAs; namely, their ability to show real-time differentiation and cellular growth kinetics between replicates within an experiment, and substantiating the viability of cells in toxicity testing assays $[37]$.

It also demonstrates where the electrode configuration and applied frequency can be chosen according to the study under investigation. Using one electrode per well detects changes within a specific location of cell culture, averaging the signal only from cells growing on that electrode, useful for detecting micromotion of cells or in wound healing assays. However, assays using wells with interdigitated multi-electrode plates give a representation of the average electrical signal across various locations within the well, which is better suited to toxicological studies.

\section{Cite this article}

Morgan K, Gamal W, Samuel K, Morley SD, Hayes PC, Bagnaninchi P, Plevris JN. Role of Impedance Based Cellular Assays in the Hepatology Research, Encyclopedia, 2020, v1, Available online:

https://encyclopedia.pub/item/revision/d14b6386981d8d00a417186d0cff22ad

\section{References}

1. Tomás García-Sánchez; Ramón Bragós; Lluis M. Mir; In vitro analysis of various cell lines responses to electroporative electric pulses by means of electrical impedance spectroscopy. Biosensors and Bioelectronics 2018, 117, 207-216, 10.1016/j.bios.2018.06.016.

2. Pierre O. Bagnaninchi; Nicola Drummond; Real-time label-free monitoring of adipose-derived stem cell differentiation with electric cellsubstrate impedance sensing.. Proceedings of the National Academy of Sciences 2011, 108, 6462-7, 10.1073/pnas.1018260108.

3. Mayyasa Rammah; Farah Dandachi; Rola Salman; Alan Shihadeh; Marwan El-Sabban; In vitro cytotoxicity and mutagenicity of mainstream waterpipe smoke and its functional consequences on alveolar type II derived cells.. Toxicology Letters 2012, 211, 220-31, 10.1016/j.toxlet.2012.04.003.

4. Moodley, K.; Angel, C.E.; Glass, M.; Graham, E.S. Real-time profiling of NK cell killing of human astrocytes using xCELLigence technology. J. Neurosci. Methods 2011, 200, 173-180.

5. Jacques-Aurélien Sergent; Vincent Paget; Sylvie Chevillard; Toxicity and Genotoxicity of Nano-SiO2 on Human Epithelial Intestinal HT29 Cell Line. Annals of Occupational Hygiene 2012, 56, 622-630, 10.1093/annhyg/mes005.

6. Ignacio Benedicto; Francisca Molina-Jiménez; Birke Bartosch; François-Loïc Cosset; Dimitri Lavillette; Jesús Prieto; Ricardo Moreno- 
Otero; Agustín Valenzuela-Fernández; Rafael Aldabe; Manuel Lopez Cabrera; et al.Pedro L. Majano The Tight Junction-Associated Protein Occludin Is Required for a Postbinding Step in Hepatitis C Virus Entry and Infection 10.1128/JVI.00038-09.

7. Mirjam B Zeisel; Punita Dhawan; Thomas F Baumert; Tight junction proteins in gastrointestinal and liver disease. Gut 2018, 68, 547561, 10.1136/gutjnl-2018-316906.

8. Jamel Mankouri; Cheryl Walter; Hazel Stewart; Matthew Bentham; Wei Sun Park; Won Do Heo; Mitsunori Fukuda; Stephen Griffin; Mark Harris; Release of Infectious Hepatitis C Virus from Huh7 Cells Occurs via a trans-Golgi Network-to-Endosome Pathway Independent of Very-Low-Density Lipoprotein Secretion.. Journal of Virology 2016, 90, 7159-70, 10.1128/JVI.00826-16.

9. Roghayeh Teimourpour; Zahra Meshkat; Aida Gholoubi; Hosein Nomani; Sina Rostami; Viral Load Analysis of Hepatitis C Virus in Huh7.5 Cell Culture System. Jundishapur Journal of Microbiology 2015, 8, e19279, 10.5812/jjm.8(5)2015.19279.

10. Bruno Sainz; Naina Barretto; Susan L. Uprichard; Hepatitis C Virus Infection in Phenotypically Distinct Huh7 Cell Lines. PLOS ONE 2009, 4, e6561, 10.1371/journal.pone.0006561.

11. Ralf Bartenschlager; Thomas Pietschmann; Efficient hepatitis $C$ virus cell culture system: what a difference the host cell makes.. Proceedings of the National Academy of Sciences 2005, 102, 9739-40, 10.1073/pnas.0504296102.

12. Ndiémé Ndongo-Thiam; Pascale Berthillon; Elisabeth Errazuriz; Isabelle Bordes; Sylvie De Sequeira; Christian Trepo; Marie-Anne Petit; Long-term propagation of serum hepatitis $\mathrm{C}$ virus (HCV) with production of enveloped HCV particles in human HepaRG hepatocytes. Hepatology 2011, 54, 406-417, 10.1002/hep.24386.

13. N. F Fletcher; A. R Clark; P Balfe; J. A McKeating; TNF superfamily members promote hepatitis $C$ virus entry via an NF-KB and myosin light chain kinase dependent pathway. Journal of General Virology 2017, 98, 405-412, 10.1099/jgv.0.000689.

14. Masato Yoneda; Jinhee Hyun; Silvia Jakubski; Satoru Saito; Atsushi Nakajima; Eugene R. Schiff; Emmanuel Thomas; Hepatitis B Virus and DNA Stimulation Trigger a Rapid Innate Immune Response through NF-KB. The Journal of Immunology 2016, 197, 630-643, 10.4049/jimmunol.1502677.

15. Fumio Kasai; Noriko Hirayama; Midori Ozawa; Motonobu Satoh; Arihiro Kohara; HuH-7 reference genome profile: complex karyotype composed of massive loss of heterozygosity. Human Cell 2018, 31, 261-267, 10.1007/s13577-018-0212-3.

16. H Nakabayashi; K Taketa; K Miyano; T Yamane; J Sato; Growth of human hepatoma cells lines with differentiated functions in chemically defined medium.. Cancer Research 1982, 42, 3858-3863.

17. Castell, J.V.; Gomez-Lechon Maria, J.; Ponsoda, X.; Bort, R.. In Vitro Methods in Pharmaceutical Research; Academic Press Limited: London, UK, 1996; pp. 375-410.

18. H. H. J. Gerets; K. Tilmant; B. Gerin; H. Chanteux; B. O. Depelchin; S. Dhalluin; F. A. Atienzar; Characterization of primary human hepatocytes, HepG2 cells, and HepaRG cells at the mRNA level and CYP activity in response to inducers and their predictivity for the detection of human hepatotoxins.. Cell Biology and Toxicology 2012, 28, 69-87, 10.1007/s10565-011-9208-4.

19. Matthew R. Pennington; Gerlinde R. Van De Walle; Electric Cell-Substrate Impedance Sensing To Monitor Viral Growth and Study Cellular Responses to Infection with Alphaherpesviruses in Real Time. mSphere 2017, 2, e00039-17, 10.1128/mSphere.00039-17

20. Lorenzo Tibaldi; Shirley Leyman; Andre Nicolas; Sofie Notebaert; Melissa Dewulf; Thu Hoa Ngo; Claudia Zuany-Amorim; Nathalie Amzallag; Isabelle Bernard-Pierrot; Xavier Sastre-Garau; et al.Clotilde Théry New Blocking Antibodies Impede Adhesion, Migration and Survival of Ovarian Cancer Cells, Highlighting MFGE8 as a Potential Therapeutic Target of Human Ovarian Carcinoma. PLOS ONE 2013, 8, e72708, 10.1371/journal.pone.0072708.

21. Hope A. Sansing; Nicole A. Renner; Andrew G. MacLean; An inverted blood-brain barrier model that permits interactions between glia and inflammatory stimuli.. Journal of Neuroscience Methods 2012, 207, 91-6, 10.1016/j.jneumeth.2012.03.015.

22. S. Kustermann; F. Boess; A. Buness; M. Schmitz; M. Watzele; T. Weiser; T. Singer; L. Suter; A. Roth; A label-free, impedance-based real time assay to identify drug-induced toxicities and differentiate cytostatic from cytotoxic effects. Toxicology in Vitro 2013, 27, 15891595, 10.1016/j.tiv.2012.08.019.

23. Zoya Marinova; Susanne Walitza; Edna Grünblatt; 5-HT2A serotonin receptor agonist DOI alleviates cytotoxicity in neuroblastoma cells: Role of the ERK pathway. Progress in Neuro-Psychopharmacology and Biological Psychiatry 2013, 44, 64-72,

10.1016/j.pnpbp.2013.01.017.

24. Moniri, M.R.; Young, A.; Reinheimer, K.; Rayat, J.; Dai, L.J.; Warnock, G.L. Dynamic assessment of cell viability, proliferation and migration using real time cell analyser system (RTCA). Cytotechnology 2015, 67, 379-386.

25. Tomás García-Sánchez; Ramón Bragós; Lluis M. Mir; In vitro analysis of various cell lines responses to electroporative electric pulses by means of electrical impedance spectroscopy. Biosensors and Bioelectronics 2018, 117, 207-216, 10.1016/j.bios.2018.06.016.

26. Katie Morgan; Nicole Martucci; Ada Kozlowska; Wesam Gamal; Filip Brzeszczyński; Philipp Treskes; Kay Samuel; Peter Hayes; Lenny Nelson; Pierre Bagnaninchi; et al.Joanna BrzeszczynskaJohn PlevrisFillip Brzeszczynki Chlorpromazine toxicity is associated with disruption of cell membrane integrity and initiation of a pro-inflammatory response in the HepaRG hepatic cell line.. Biomedicine \& Pharmacotherapy 2019, 111, 1408-1416, 10.1016/j.biopha.2019.01.020.

27. Franck A. Atienzar; Karen Tilmant; Helga H. Gerets; Gaëlle Toussaint; Sebastien Speeckaert; Etienne Hanon; Olympe Depelchin; Stéphane Dhalluin; The Use of Real-Time Cell Analyzer Technology in Drug Discovery. Journal of Biomolecular Screening 2011, 16, 575-587, 10.1177/1087057111402825.

28. Franck André Atienzar; Helga Gerets; Karen Tilmant; Gaëlle Toussaint; Stephane Dhalluin; Evaluation of Impedance-Based Label-Free Technology as a Tool for Pharmacology and Toxicology Investigations. Biosensors 2013, 3, 132-156, 10.3390/bios3010132. 
29. Ludovic Peyre; Georges De Sousa; Sylvie Barcellini-Couget; Anne-Pascale Luzy; Nathalie Zucchini-Pascal; Roger Rahmani; Highcontent screening imaging and real-time cellular impedance monitoring for the assessment of chemical's bio-activation with regards hepatotoxicity. Toxicology in Vitro 2015, 29, 1916-1931, 10.1016/j.tiv.2015.07.024.

30. Wesam Gamal; Philipp Treskes; Kay Samuel; Gareth J. Sullivan; Richard Siller; Vlastimil Srsen; Katie Morgan; Anna Bryans; Ada Kozlowska; Andreas Koulovasilopoulos; et al.lan UnderwoodStewart SmithJorge Del-PozoSharon MossAlexandra Inés ThompsonNeil C. HendersonPeter C. HayesJohn N. PlevrisPierre-Olivier BagnaninchiLeonard J. Nelson Low-dose acetaminophen induces early disruption of cell-cell tight junctions in human hepatic cells and mouse liver. Scientific Reports 2017, 7, 37541, 10.1038/srep37541.

31. Philippe Gripon; Sylvie Rumin; Stephan Urban; Jacques Le Seyec; Denise Glaise; Isabelle Cannie; Claire Guyomard; Josette Lucas; Christian Trepo; Christiane Guguen-Guillouzo; et al. Infection of a human hepatoma cell line by hepatitis B virus. Proceedings of the National Academy of Sciences 2002, 99, 15655-15660, 10.1073/pnas.232137699.

32. Marc Le Vée; Elodie Jouan; Bruno Stieger; Olivier Fardel; Differential regulation of drug transporter expression by all-trans retinoic acid in hepatoma HepaRG cells and human hepatocytes. European Journal of Pharmaceutical Sciences 2013, 48, 767-774, 10.1016/j.ejps.2013.01.005.

33. Monika Szabo; Zsuzsa Veres; Zsolt Baranyai; Ferenc Jakab; Katalin Jemnitz; Comparison of Human Hepatoma HepaRG Cells with Human and Rat Hepatocytes in Uptake Transport Assays in Order to Predict a Risk of Drug Induced Hepatotoxicity. PLOS ONE 2013, 8, e59432, 10.1371/journal.pone.0059432.

34. Yong Soon Chun; Pooja Chaudhari; Yoon-Young Jang; Applications of Patient-Specific Induced Pluripotent Stem Cells; Focused on Disease Modeling, Drug Screening and Therapeutic Potentials for Liver Disease. International Journal of Biological Sciences 2010, 6, 796-805, null.

35. Gareth J. Sullivan; David C. Hay; In-Hyun Park; Judy Fletcher; Zara Hannoun; Catherine M. Payne; Nna Dalgetty; James R. Black; James A. Ross; Kay Samuel; Gang Wang; George Q. Daley; Je-Hyuk Lee; George M. Church; Stuart J. Forbes; John P. Iredale; lan Wilmut; Generation of functional human hepatic endoderm from human induced pluripotent stem cells.. Hepatology 2010, 51, 329-35, 10.1002/hep.23335.

36. S. Tamir Rashid; Graeme J.M. Alexander; Induced pluripotent stem cells: From Nobel Prizes to clinical applications. Journal of Hepatology 2013, 58, 625-629, 10.1016/j.jhep.2012.10.026.

37. Ying Zhou; Dahou Yang; Yinning Zhou; Bee Luan Khoo; Jongyoon Han; Ye Ai; Characterizing Deformability and Electrical Impedance of Cancer Cells in a Microfluidic Device. Analytical Chemistry 2017, 90, 912-919, 10.1021/acs.analchem.7b03859.

\section{Keywords}

impedance; hepatology; gastroenterology

(C) 2020 by the author(s). Distribute under aCreative Commans CC BY license 